\title{
2 Categorizing Ideas about Systematics: Alternative Trees of Trees, and Related
}

\section{Representations}

4 János Podani ${ }^{1}$, David A. Morrison ${ }^{2}$

51 Department of Plant Systematics, Ecology and Theoretical Biology, Institute of Biology,

6 Eötvös University and Ecology Research Group of the Hungarian Academy of Sciences,

7 Budapest, Hungary. Corresponding author. Email: podani@ludens.elte.hu, Telephone: +36 1

$8 \quad 3812293$

92 Systematic Biology, Evolutionary Biology Center, Uppsala University, Uppsala, Sweden.

10 E-mail: david.morrison@ebc.uu.se

11 Acknowledgements. We thank Ferenc Jordán for preparing the diagram of the plexus graph.

12 We are grateful to the anonymous referees for the constructive criticism of the manuscript.

13 Abstract. This study is an attempt to expand a previous survey by Fisler and Lecointre (FL)

14 for systematizing ideas on the use of the tree metaphor in classification, as expressed by

15 various historically important figures in their writings. FL used a cladistic approach to analyze

16 their data, as employed in biological classification. We supplement this analysis here using

17 several methods of multivariate data exploration, producing a UPGMA dendrogram, a

18 minimum spanning tree, a neighbor joining additive tree, a plexus graph, a phylogenetic

19 network, and two multidimensional scaling ordinations of the same data used by FL. We

20 confirm the validity of many of FL's smaller clusters of writings, and revealed a new 3-group

21 categorization undetected by the previous study. These three groups largely correspond to

22 Classifiers, who did not consider evolution for historical reasons or on purpose, Non-

23 analytical evolutionists, who recognized evolution but with a more or less naïve attitude

24 towards the temporal change of life, and Modelers, with more explicit views on evolutionary 
25

26

27

28

29

30

31

processes, often applying objective mathematical tools for exploring the past and present of organismal diversity. Some scientists were difficult to assign to any group unambiguously, including J.W. von Goethe, who takes a unique position in the history of biology, and, to a lesser extent, E. Mayr and G.G. Simpson, the leaders of the gradist school of systematics. We argue that cladistic methods are insufficient by themselves, notably in situations where there are no obvious ancestor-descendant relationships underlying the development of the objects being analyzed.

Keywords: Classification, Evolutionary theory, Cladistics, Network, Ordination.

\section{Introduction}

In historical accounts of science, it is a common practice to assign labels to members of various intellectual schools, such as Pythagoreans, Essentialists, Darwinists, or Popperians. These categories are determined in most cases based on a fairly subjective basis, without formal quantitative analyses of the views expressed by designated representatives of these schools. A noted exception is a recent paper by Fisler and Lecointre (2013; abbreviated hereafter as FL) who recognized that ideas in biology may be described in terms of many measurable variables simultaneously, allowing the possibility of objective comparisons.

FL selected 41 published works with the purpose to categorize ideas about "phylogenetic" trees and tree-based classifications. These writings encompass several centuries of scientific advancement, from A. Zaluziansky who worked late in the sixteenth century to P. Tassy whose ideas were summarized at the end of the twentieth century. Both written text and drawings were evaluated for 91 different variables that conceptualize the ideas being expressed. The resulting $41 \times 91$ data matrix was analyzed by the cladistic method of 
maximum parsimony (rooted in the works of Hennig 1966), in order to reveal "clades"1 of authors and to see how the different ideas were shared by their proponents in these groups. All conclusions were based on a hierarchical arrangement, a so-called "tree of trees", generated as a single, unweighted consensus cladogram of 279 equally parsimonious results. The authors were able to identify well-known schools of systematics, and they also specified some groups that are apparently new to the history of biology.

We find that whereas their pioneering approach is extremely interesting and thoughtprovoking, it is remarkably one-sided. The cladistic analysis is used, in fact, for classificatory purposes, rather than for other possible relationships among the objects, such as direct ancestry or inter-object dissimilarity evaluated without imposing cluster structure on the data. However, many other methods are widely available in the statistical literature, for both classification and other forms of relationship. The cladistic method adopted belongs to one particular school of systematics, although other schools have also employed objective procedures for tree-making. Cladistic methods have been used to reveal relationships for objects with an evolutionary history, such as organisms (Hennig 1966; and his followers), languages (Rexová et al. 2003), archaeological specimens (O’Brien et al. 2001), music (Le Bomin et al. 2015) or even biblical scripts (Howe and Windram 2011). The fact that cladistics does not well fit the complex development of biological thought is admitted by Fisler and Lecointre themselves, who said that "the flow of ideas through times doesn't behave like in biological entities". Similarities between ideas are obviously not due to simple "inheritance" or "ancestry" and therefore cladograms may not be the best approach, and are definitely not the only appropriate representations of quantifiable structure in the data.

\footnotetext{
${ }^{1}$ Here we use quotation marks, because clade is generally understood as a group of objects with common ancestry (monophyly) whereas in FLs study "clades" do not necessarily satisfy this requirement. These are groups formally optimized by a cladistic method.
} 
70 Phenetics, another school of systematics covered by Fisler and Lecointre's study, however,

71 uses a much wider range of procedures for evaluating similarities, revealing categories as well

72 as visualizing results in various graphical forms, such as networks, dendrograms and

73 ordinations. There are also network-generating procedures, which do have applications in

74 phylogenetics and elsewhere (Morrison 2014), whose capabilities were explicitly ignored by

75 FL. Other tree-generating methods and ordination procedures are effective summaries of

76 multivariate data, but as such they will differ from each other depending on which aspects of

77 the data are emphasized in the summary. These methods are therefore usually complementary,

78 in that when they are considered together they can reveal patterns that are not necessarily

79 obvious in any one data summary. So, it is best to use a combination of clustering, network

80 and ordination methods in order to thoroughly explore any given multivariate data set.

81 Our approach here is explicitly one of exploratory data analysis (Tukey 1977). This

82 methodology eschews the idea of testing formal hypotheses that can be stated a priori, but

83 instead explores the data in a model-independent manner. Graphical representations of the

84 data are an important part of data exploration (Ellison 2001), rather than formal statistical

85 analyses. Exploratory data analysis is useful in any field of science, from anthropology

86 through psychology to zoology, including phylogenetics (Morrison 2010), in which many

87 objects are described in terms of many features or variables.

88 It is important to note that time is not explicitly incorporated into any of the multivariate

89 analyses, not even cladistics. The data are analyzed to display patterns of similarity among the

90 objects, and at least some of these patterns will reflect the history of the objects, but not

91 necessarily in any explicit way. So, the fact that Ernst Mayr and Willi Hennig, for example,

92 might have been familiar with the ideas of Charles Darwin, but not vice versa, is irrelevant to

93 the analyses - all of the studied works are treated as equal. 
94 The primary objective of the present paper is to demonstrate that this approach is equally

95 applicable to humanities (e.g., Behrens and Yu 2003), including the historical sciences. We

96 show that the simultaneous use of alternative procedures of exploratory data analysis may

97 provide different insights into the same problem. In this way, we are able to reveal a pattern

98 that was not disclosed by FL, and thus showing future directions towards an even more

99 objective and meaningful evaluation of the history of thought in the biological sciences.

1002 Methods

101 In the present study, we use exactly the same data as used in Fisler and Lecointre (2013, their

102 Table 3): 41 works (OTUs ${ }^{2}$ ) described in terms of 91 variables, all of them nominal, with

103 mostly 2 , or rarely 3 , states (possible values). Nominal variables represent the simplest type of

104 data we can have: by using them the only judgment we can make about the OTUs is whether

105 or not they possess the given variable. Ordering and differences between the possible states of

106 the variable convey no meaning whatsoever. For example, in a given tree diagram drawn by

107 some biologist the vertical axis may correspond to time (coded by 1 ) or not (coded by 0 ), as

108 expressed by variable 38 of FL.

109 Our approach is dissimilarity-based, which means that the OTUs are compared in every

110 possible pair by an appropriate mathematical function. The literature abounds in such

111 measures, but in the present case our choice was limited: the data set contained many

112 irrelevant or missing scores (there were $788=21 \%$ such entries in the matrix used by FL),

113 which cannot be handled by most dissimilarity measures. We therefore used the Gower

\footnotetext{
${ }^{2}$ In the terminology of numerical taxonomy, an OTU (= "operational taxonomic unit") represents an individual study object, in our case a specified scientific writing due to a specified author. Each OTU appears as a single vertex (or node) in tree-like diagrams or as a point in ordination scatter plots.
} 
114 (1971) formula which can also handle variables of the nominal type. The formula takes the

115 following form:

$116 \mathrm{~d}_{\mathrm{jk}}=\frac{\sum_{\mathrm{i}=1}^{\mathrm{n}} \mathrm{w}_{\mathrm{ijk}} \mathrm{a}_{\mathrm{ijk}}}{\sum_{\mathrm{i}=1}^{\mathrm{n}} \mathrm{w}_{\mathrm{ijk}}}$,

117 where $\mathrm{n}$ is the number of variables, $\mathrm{a}_{\mathrm{ijk}}=0$ if OTUs $\mathrm{j}$ and $\mathrm{k}$ agree in variable $\mathrm{i}$, and $\mathrm{a}_{\mathrm{ijk}}=1$

118 otherwise. Weight $\mathrm{w}_{\mathrm{ijk}}=1$ if OTUs $\mathrm{j}$ and $\mathrm{k}$ are comparable for variable $\mathrm{i}$, and $\mathrm{w}_{\mathrm{ijk}}=0$ if either

119 or both OTUs have a missing or undefined score for that variable. The dissimilarity values

120 have the range from zero to unity, 0 meaning complete identity and 1 referring to maximum

121 dissimilarity.

122 All pairwise comparisons yielded a $41 \times 41$ dissimilarity matrix of OTUs, which was the

123 starting point for all subsequent analyses, to produce a phenetic dendrogram (UPGMA

124 clustering), a minimum spanning tree and a rooted additive tree (neighbor joining), a plexus

125 graph and a phylogenetic network (neighbor net), and two ordinations (multidimensional

126 scaling). Some comments on each of these methods will be given in the Results section,

127 where the reader is referred to the cited literature on multivariate analysis and systematics for

128 more details. The diagrams thus obtained are compared with each other and with the FL

129 cladogram (called Tree 1 in this paper; Figure 1) in order to determine whether: i) the

130 cladogram nodes they recognized as meaningful indicators of groups (or schools, alternative

131 approaches) are corroborated, and ii) any new information is also recovered from the data.

132 It is also important to note that these analyses place OTUs as sisters to each other, rather than

133 placing some of them as ancestors and descendants, as would be true in an explicitly time- 
134 constrained analysis. It is impossible to determine from the data whether one OTU is the

135 ancestor of another, and so they are placed in sister-group relationships.

136 Zaluziansky and Linnaeus are handled in the same manner as every other historical figure in

137 all but one of the analyses. The exception is neighbor joining, in which Zaluziansky took a

138 special position as an "outgroup" (see details below). Calculations were made using the SYN-

139 TAX 2000 package (Podani 2001), except for the plexus graph drawn by the UciNet software

140 (Borgatti et al. 2002) and the phylogenetic network computed by SplitsTree 4 (Huson and

141 Bryant 2006).

1423 Results

\section{$143 \quad 3.1$ Tree 2: dendrogram}

144 The phenetic alternative to conventional cladograms is the dendrogram, which converts

145 dissimilarities to ultrametric distances (Lapointe and Legendre 1995). We used the group

146 average (or UPGMA ${ }^{3}$, Sneath and Sokal 1973) algorithm for clustering, because it is also

147 well-known in phylogenetic systematics, as a standard distance-based tree generating routine

148 (meaningful whenever the molecular clock is "on"; Swofford et al. 1996; Page and Holmes

149 1998) and has been the most extensively used clustering procedure in many areas of science

150 outside biology. For example, Babitch and Lebrun (1989) used this method for classifying

151 languages and dialects, while Prieto et al. (2014) compared archaeological findings, namely

152 terra-cotta figurines, by UPGMA. A dendrogram may be interpreted as a series of partitions

153 (i.e., classifications into disjoint sets) in which small subsets (groups or clusters) are

154 successively nested within large ones. The dendrogram may be "cut" at a given level to obtain

155 a partition set.

\footnotetext{
${ }^{3}$ Unweighted pair-group method using arithmetic averages.
} 
156 Here, we recognized a partitioning into three major clusters (Figure 2, see also the Electronic

157 Appendix), none of them in complete agreement with the "clades" in FL. In cluster A

158 ("Classifiers"), which is the first one separated from the rest, we find tree users from the pre-

159 evolutionary age of biology, plus some later authors who deliberately created a tree-based

160 classification without evolutionary considerations (Wallace56 and Richenow, node 75 in FL).

161 Goethe does not belong to this cluster, because he forms a singleton group, if we cut the tree

162 around the dissimilarity level of 0.39 . This reflects the ambiguity in his controversial views on

163 "metamorphosis", a fact still subject to intensive debate among historians of evolutionary

164 biology (see e.g., Richards 2015; Spahn 2015). The special position of Goethe among the

165 writers evaluated here is confirmed by the fact that in order to encounter the next singleton

166 cluster (Haeckel66) one has to move down to a dissimilarity of 0.26 . Note that on the FL

167 cladogram, Goethe was also uniquely positioned.

168 Clusters B and C together (=FL node 73) include almost exclusively authors and works that

169 recognized evolution, with different emphasis on its various aspects. The only exception is

170 perhaps Agassiz, a believer in creation, whose presence in cluster B is due probably to the fact

171 that he arranged fossils on the tree according to geological time. Cluster B unites the large

172 group of metaphoricians (FL node 60) with Buffonians (FL node 44) and gradists (Mayr53,

173 Mayr82 and Simpson - the latter two being FL node 66 as "grade theoreticians"). It is

174 reasonable to call this large group collectively as "Non-analytical evolutionists" because

175 subjective judgment had a primary role in their thinking about systematics. In their views,

176 classifications enjoyed in most cases priority and evolution was considered only later to

177 explain the classification.

178 Cluster C, on the other hand, comprises "Modelers", who explicitly used trees to demonstrate

179 evolutionary processes (Wallace and Darwin) or computed the tree to provide a starting basis 
180 for an a posteriori classification (cladists, FL node 63, and pheneticists, FL node 68). The

181 relative closeness of cladists and pheneticists in Tree 2 may be surprising to some people, but

182 they agree in many features, especially in their ambition to place biological classification on

183 objective foundations, both theoretically and empirically. Also, the complex association of

184 Haeckel, Darwin and Lamarck in the analyses is interesting, because both Haeckel and

185 Lamarck saw evolution as an inherently progressive process, whereas Darwin did not.

186 All of the seven Classifiers are entirely homogeneous for five characters (emphasized by

187 rectangles in the Electronic Appendix): 43 (state 0, the tree has classificatory aim), 44 (0,

188 classification not made before the tree), $49(0$, the tree is not genealogical), $60(0$, time not

189 considered) and 83 ( 0 , no parsimony) - the latter two are also true for Goethe as well. There is

190 no character state which would exclusively occur here. The relatively large group of Non-

191 analytical evolutionists has only a single homogeneous variable, 83 ( 0 , no parsimony).

192 However, character states that predominate in this group, with no more than 3 exceptions or

193 missing values, include: 1 ( 0 , concrete ancestor at the root), 2 ( 0 , no initial character states at

194 the root), 3 ( 0 , no inorganic forms included), 13 ( 0 , no conceptual nodes), 35 ( 0 ,

195 diversification axis carries no time), 47 (1, Nature is fundamentally ordered), 48 ( 0 , tree is

196 explicit), 51 (1, gradation in perfection), 72 (0, groups are not made according to genealogical

197 links), 76 (1, groups are linked or nested), 84 (0, classification includes lack of shared

198 properties), 86 (0, classification by global similarity) and 90 ( 0 , homoplasies cannot be

199 detected). Overall, cluster C, the Modelers, are the most homogeneous: they completely agree

200 in 22 variables, and in a further 17 if Wallace55 is not considered (therefore the long list of

201 variables is not given here). Not surprisingly, parsimony is the only character state that occurs

202 exclusively in cluster C. See the Electronic Appendix for these character distributions. 
204 This tree connects OTUs directly such that the sum of weights assigned to the links (i.e.

205 dissimilarities in this case) is the minimum (Rohlf 1973). It follows that terminal objects are

206 always linked to their nearest neighbors. Successive removal of the longest links produces a

207 hierarchical classification identical to the single-link clustering result. This type of tree was

208 occasionally used in the initial period of numerical cladistics as a starting graph for phylogeny

209 reconstruction. A noted example of using such trees outside biology is provided by Hage et al.

210 (1996) in archaeology. In general, it serves as an alternative display of relationships to

211 confirm or reject hypotheses of topological relationships. In our case, the overall arrangement

212 of OTUs (Figure 3) reflects quite well the clusters of Tree2: the three groups are easily

213 distinguishable along the main axis of the tree, which represents the longest path, that

214 between Linnaeus and Sokal66. The largest dissimilarity separates group A from B

215 (connecting Rühling with Barbançois16a), while the second longest edge separates Goethe

216 from his nearest neighbor, Richenow, confirming the ambiguity of categorizing Goethe's

217 writings. Apparently, Mayr62 and Simpson, the theoretical gradists in FL, represent a

218 somewhat transitional position between Non-analytical evolutionists (B) and Modelers (C).

219 Buffon and Duchesne form their own subtree, the Buffonians (comparable with FL node 44).

220 The metaphoricians (FL node 60) take the central position, from Barbançois16b to Agassiz.

221 That is, in many details this tree agrees fairly well with Tree 1 as well.

\section{$222 \quad 3.3$ Tree 4: additive tree}

223 The objective here is to generate a tree in which the between-object dissimilarities are as close

224 as possible to the dissimilarities in the input matrix, and so clusters are not optimized directly.

225 In this sense, this construct, most easily computed by the neighbor joining algorithm (Saitou

226 and Nei 1987), is conceptually closest to cladograms, and it is often used in phylogenetics

227 when the input matrix represents meaningful evolutionary distances. It has also been 
228 recommended as an adequate representation of manuscript traditions (Najock 1989). The

229 algorithm produces an unrooted tree, which may be rooted by designating one OTU as the

230 outgroup (here Zaluziansky) for comparability with rooted cladograms and dendrograms. As

231 seen (Figure 4), Linnaeus is very close to Zaluziansky, justifying the decision of FL to select

232 both of them as outgroups in parsimony analysis. Since it is not a clustering method, the large

233 UPGMA groups are broken into parts that separate from the rest one by one as we proceed

234 farther and farther from the root. The classifiers appear in two subtrees, with Goethe linked to

235 Richenow and Wallace56 (see Tree 3). It is remarkable that pheneticists (FL node 68) are

236 separated from the strictly genealogical classifiers (FL node 65, from Wallace55 to Tassy),

237 with some classifiers (FL node 78, e.g., Augier) and two gradists in between. This

238 arrangement confirms the earlier findings that Mayr82 and Simpson are in a fairly equivocal

239 position. The additive tree agrees with Tree 1, in that the Buffonians form a separate "clade"

240 and that the metaphoricians (FL node 60) appear as an intact group.

\section{$241 \quad 3.4$ Plexus graph}

242 A conventional network graph differs from the minimum spanning tree in that there may be

243 several different paths between two OTUs, i.e., there can be circular paths. Such graphs have

244 been extensively used in the historical sciences (Gould 1993) and in citation analysis (Cronin

245 and Atkins 2000). An example relevant to our study is provided by Krischel and Fangerau

246 (2013), who compiled a social network for nineteenth century evolutionists, anthropologists

247 and linguists, in which node size was determined by connectedness - Darwin's node being the

248 largest (their Figure 5). Such a graph is not appropriate here, however, because the

249 relationship between writers is not of the yes-or-no type, but instead is measured on a

250 continuous scale. 
251 Therefore, we used a plexus graph in which the edges are drawn with different thickness (or

252 color) depending on the dissimilarity between pairs of OTUs, a tool favored in the pioneering

253 age of numerical ecology (McIntosh 1978). Here, we decided to use categories of

254 dissimilarities, which are usually sufficient to reveal "coalitions" among the OTUs. These

255 categories are, in decreasing order of line thickness: $0 \leq d<0.1 ; 0.1 \leq d<0.2 ; 0.2 \leq d<0.3$,

$2560.3 \leq d<0.4$. Pairs of writers with a dissimilarity of $d \geq 0.4$ are not connected. The OTUs

257 were arranged in a plane using the spring embedding algorithm. The plexus graph thus

258 obtained confirms the existence of the three major groups recognized above (Figure 5). Most

259 of the thickest edges connect members of Modelers (group C), which are associated to the

260 Non-analytical evolutionists (group B) through weaker links $(0.2 \leq d<0.3)$, with the

261 exception of the connection between Wallace55 and Simpson. The cohesion within the Non-

262 analytical evolutionists is weaker, whereas connectedness is fairly high, as it is within the

263 Classifiers (group A). In the latter, Zaluziansky and Linnaeus, as well as Wallace56 and

264 Richenow form close pairs. Duchesne and Augier represent the transition between Buffonians

265 and the Classifiers. Note the central position of Mayr53, with links to all the three groups, and

266 that of Goethe, who is apparently an outlier in the system.

\subsection{Phylogenetic network}

In addition to plexus graphs, there are many other types of networks used in biology. Those of

269 particular interest here combine the hierarchical grouping properties of the clustering methods

270 (see above) with the spatial representation of ordinations (see below) (Morrison 2014). These

271 so-called "phylogenetic network" methods are increasing in popularity because they help test

272 whether the data contain a strong tree-like signal, and will display a set of overlapping

273 clusters if they do not. Note that the plexus network connects the OTUs via observed links,

274 while phylogenetic networks connect them via inferred links and inferred nodes. The latter 
275 networks may be either more or less complex than the former. The use of such networks is by

276 no means restricted to evolutionary biology (see Morrison 2014, for examples from other

277 fields such as stemmatology, linguistics and archaeology). The main conceptual difference

278 from trees is that trees produce nested groups whereas networks produce overlapping (i.e.

279 non-exclusive) groups.

280 The neighbor net method, used here, starts from a dissimilarity matrix directly, producing a

281 planar representation of the multivariate patterns. The resulting network (Figure 6)

282 successfully displays $88.9 \%$ of the information in the original distance matrix. This is not a

283 very tree-like network, indicating that the tree-based methods may be over-interpreting the

284 groupings of the OTUs. Indeed, the phylogenetic network has more similarity to the

285 ordination diagrams (see below) than to the trees (see above). The Classifiers and Modelers

286 can be readily separated, but the Non-analytical evolutionists form a grade between them, as

287 in Tree 3 (Figure 3), with the Buffonians distinct from the rest. Goethe has a long terminal

288 edge, as expected to indicate his equivocal position, but the gradists do not have an especially

289 marginal position in the network. On the other hand, the three works by Haeckel are not

290 closely associated in the network, which they are in the trees and also to some extent in the

291 ordinations - this seems to reflect the complex patterns of missing data for these three

292 works.

\section{$293 \quad 3.6$ Ordinations}

294 As a supplementary tool for the line-graph representations, it is always worth trying some

295 methods of ordination to reduce dimensionality in the original data space into a few axes

296 (represented as a scatter plot), and then to evaluate whether clusters are distinguishable along

297 these dimensions (Podani 2000). If the data set has a meaningful pattern because the original

298 variables are correlated, then 2-3 ordination axes may be sufficient to display the inter-point 
299 relationships with a negligible loss of information. The "success" of the axes is expressed in

300 terms of the percentage of eigenvalues of the starting matrix. Ordinations have been rarely

301 used for phylogenetic purposes, but they are common in other fields of biology such as

302 ecology, as well as in the archaeological sciences (Hodson et al. 1971). Since our raw data

303 include too many missing values, only one group of ordination procedures is applicable here,

304 namely multidimensional scaling, as these methods start from a dissimilarity matrix directly,

305 in our case from the Gower dissimilarities.

306 We first used Principal coordinates analysis (PCoA), a metric procedure which arranges the

307 OTUs in a new coordinate system such that the inter-point dissimilarities reproduce the

308 original dissimilarities. Although no compact groups of OTUs are indicated (Figure 7), the

309 arrangement of points is in complete harmony with the groups in Tree2. Classifiers, Non-

310 analytical evolutionists and Modelers can be readily separated by straight lines in the first two

311 dimensions. Goethe falls far from all other writers in the scatter plot, while the gradists

312 Simpson and Mayr53 (but interestingly not Mayr82) take a marginal position in the group of

313 Non-analytical evolutionists. Minor groups, such as pheneticists and cladists, are clear-cut in

314 the diagram. The first eigenvalue explains $27.1 \%$ of the total variance, while the second one

315 accounts for a further $21.6 \%$, which at first glance suggests high explanatory power in these 2

316 dimensions. However, due to the often large and varying numbers of missing scores in the

317 pairwise comparisons, there are many negative eigenvalues, with a total cumulative variance

318 approximating $20 \%$ of the sum of positive eigenvalues.

319 The appearance of negative eigenvalues in the PCoA solution is indicative of the absence of

320 true metric structure in the data, and the results may be doubtful in such cases. Thus,

321 nonmetric multidimensional scaling is called for to confirm the picture obtained by PCoA.

322 This arranges the OTUs in a pre-specified number of dimensions (usually two, representing a 
323 plane) such that the rank order of interpoint distances in the ordination is as close as possible

324 to the rank order of the original dissimilarities. The analysis is iterative, by optimizing a

325 random starting configuration; and the success of fit of the two rank orders is measured by the

326 stress function, ranging from 0 to 1 . We ran the program 20 times, and obtained the best result

3273 times, with a stress of 0.207 - which is reasonable for 41 OTUs. The ordination (Figure 8)

328 agrees with the PCoA result remarkably well suggesting that the lack of metric properties

329 does not influence our conclusions regarding the groups. The major groups may be

330 recognized as above, with Goethe isolated as always, and the gradists are again in a marginal

331 position.

\section{Discussion}

333 This study used the same data as Fisler and Lecointre (2013; FL), although we do not agree

334 completely with their selection of either scientists or characters. While most authors were

335 represented only once, several others appeared twice or even three times in the FL study. This

336 produced redundancy for those authors whose views did not vary much through time,

337 especially for Darwin and Sokal \& Sneath, and to some extent for Romer, Barbançois and

338 Teilhard de Chardin as well. On the other hand, many important contributors to the history of

339 systematics who also suggested or produced tree, tree-like or network summaries of their

340 classifications were overlooked. To mention a few: Pax, Naudin, Herdman, Bessey, Hallier,

341 Takhtajan, Whittaker, Cronquist, Doolittle and Cavalier-Smith - along with the entire school

342 of pattern cladistics. The 91 selected characters are not optimal either. Due to missing scores,

343 eight of them were not meaningful for more than 20 scientists, while three writings had

344 undefined characters for more than 50 of the variables. Some variables were redundant, while

345 none of them expressed the important distinction between a tree (as in Hennig) and a network 
346 (as in Buffon), for example. The dataset could thus be improved, although this would require

347 a considerable amount of careful extra work.

348 However, to allow a direct comparison with the results of FL and to demonstrate the utility of

349 other exploratory methods, we decided not to introduce changes. In one sense it is thus good

350 news that our results confirmed several findings made by Fisler and Lecointre (2013),

351 especially regarding the choice of outgroups, and the presence of minor "clades". Not

352 surprisingly, our Tree 4, the additive one (which may also be conceived as a distance based

353 cladogram, i.e. a phylogram), agrees the best with Treel (the FL cladistic tree) by being able

354 to detect identical "clades": initial tree users (node 78), tree makers (79), cladists (63),

355 pheneticists (68), Buffonians (44), metaphoricians (60) and strictly genealogical classifiers

356 (65). Tree 2 also shows three of these nodes, but not nodes 79,44 or 60 , while also

357 reproducing the grade theoreticians (FL node 66). Of the nodes recognized and discussed by

358 FL, the evolutionists (72) and connected graph users (70) are not reproduced by our analyses,

359 mostly due to the "misclassification" or displacement of a few writings only. Also, the group

360 of similarity classifiers $(69=66+68)$, which appears so clear-cut in Tree 1 , is refuted by all of

361 our diagrams.

362 The overall picture of the data structure differs in our analyses compared to FL, however.

363 Most of our results suggest and others confirm - or do not refute at least - the observation that

364 the scientific writings may be categorized into three separable, though not overly compact

365 groups. There are some transitions between these groups, and also people who fit into more

366 than one group. This picture is definitely more realistic than a single categorization since

367 scientific ideas are never developed in isolation, all authors may influence the works of later

368 authors, some concepts are inherited by new schools, others revised and still others 
369 completely reformulated. In other words, there is considerable fuzziness in the data which is

370 best revealed by alternative approaches.

371 Regarding historical time of first appearance, the group of Classifiers includes authors who

372 were not (yet) influenced by evolutionary theory in making their classifications or trees (such

373 as Linnaeus and Augier) or who deliberately ignored evolutionary considerations, such as

374 Wallace, who is otherwise considered together with Darwin as the developer of the theory of

375 evolution through natural selection. The second group, Non-analytical evolutionists comprises

376 authors who first recognized the existence of temporal change in organismal life, from Buffon

377 through Lamarck to Romer. Even Agassiz is here, because he recognized that the fossil record

378 changes through time, even though he was not an evolutionist. Gradists take a marginal

379 position in this group, with weak affinities to the third group. In this third group, the

380 Modelers, evolutionary change is explained by theoretical models, and its pathways are

381 reconstructed or its results are evaluated by objective methodology. That is, Darwin and

382 Wallace are not too far from Hennig conceptually, and, despite some philosophical

383 differences, they are fairly close to the school of numerical taxonomy as well.

384 Goethe is certainly a unique thinker, an "outlier" - without having a close relationship to any

385 of these groups. Notwithstanding the difficulties with the choice of data, we suggest that the

386 three-group classification of scientists is a meaningful summary of tree-thinking in biological

387 classification. Additional studies, with an expanded set of writings and more variables

388 involved, may provide further insight into and a deeper understanding of that history.

389 The present study supports the general view that for the evaluation of complex data without

390 obvious a priori structure, such as the dataset used here, the combination of various

391 multivariate techniques may extract much more information than can any one analysis alone.

392 An advantage of using alternative methods is that details supported by most procedures may 
393 be considered as "valid" structural properties of the data, such as the existence of many small

394 clusters of writings in this study. Furthermore, in this way the limitations of one procedure

395 may be compensated for by another. Clusters that appeared fairly distinct in the UPGMA

396 dendrogram, for example, proved to be less clear cut in the networks and the ordinations.

397 Although Fisler and Lecointre (2013) were skeptical about the usefulness of networks for

398 demonstrating changes of biological thought, we found them to be as meaningful as any tree

399 or ordination scatter plot.

400 We have thus shown that a purely cladistic approach to a classification problem, in which

401 historical factors play little or no role, may be supplemented effectively by the joint

402 application of various tree- and network-generating methods as well as ordinations, all of

403 which are absolutely free from the assumptions of cladism.

404 Neither the cladistic method nor any of our alternative analyses are explicitly historical —

405 historical patterns will be included in the outcome but they will not necessarily be separable

406 from patterns resulting from any other source. In this paper, we have addressed whether the

407 groups of people are robust by using different methods (i.e., the patterns are model

408 independent), but we have not explicitly tested whether they have historical meaning. We

409 have thus set up a series of hypotheses (the groups), and we have suggested possible historical

410 interpretations of these groups, and so these hypotheses can now be examined in more detail

411 and formally tested. The latter is beyond our brief, however.

412 Identifying the specifically historical pattern is, of course, important, but this goes beyond the

413 capabilities of any multivariate analysis. A much more detailed assessment of the data would

414 be required, which could now be based on the preliminary hypotheses presented here. This

415 would include more than solely mathematical analyses, such as a detailed evaluation of the 
416 context of the individual writings studied here, perhaps with the inclusion of an expanded set

417 of writings, and even then this may not be achievable with this type of intellectual inquiry.

\section{References}

419 Babitch RM, Lebrun E (1989) Dialectometry as computerized agglomerative hierarchical

$420 \quad$ classification analysis. Journal of English Linguistics 22:83-90

421 Behrens JT, Yu CH (2003) Exploratory data analysis. In: Schinka JA, Velicer WF (eds)

422 Handbook of Psychology, vol. 2: Research Methods in Psychology. John Wiley \&

423 Sons, Hoboken (NJ), pp. 33-64

424

426

427

428

429

430

431

432

434

435

436
Borgatti SP, Everett MG, Freeman LC (2002) Ucinet for Windows: Software for Social Network Analysis. Analytic Technologies, Harvard, MA

Cronin B, Atkins H B (2000) (eds). The Web of Knowledge: A Festschrift in Honor of Eugene Garfield. Information Today, Inc., Medford, NJ

Ellison AM (2001) Exploratory data analysis and graphic display. In: Scheiner SM, Gurevitch J (eds) Design and Analysis of Ecological Experiments, 2nd ed. Oxford University Press, Oxford, pp. 37-62

Fisler, M. and Lecointre, G. 2013. Categorizing ideas about trees: A tree of trees. PLOS One 8(8): e68814

Gould RV (1993) Collective action and network structure. American Sociological Review 58:182-96

Gower JC (1971) A general coefficient of similarity and some of its properties. Biometrics $27: 857-872$ 
437 Hage P, Harary F, James B (1996) The minimum spanning tree problem in archaeology.

$438 \quad$ American Antiquity 61:149-155

439 Hennig EHW (1966) Phylogenetic Systematics. University of Illinois Press, Urbana

440 Hodson FR, Kendall DG, Tăutu P (eds) (1971) Mathematics in the Archaeological and

441 Historical Sciences. Edinburgh University Press, Edinburgh.

442 Howe CJ, Windram HF (2011) Phylomemetics - evolutionary analysis beyond the gene.

$443 \quad$ PLoS Biology 9: e1001069

444 Huson DH, Bryant D (2006) Application of phylogenetic networks in evolutionary studies.

$445 \quad$ Molecular Biology and Evolution 23:254-267

446 Krischel M, Fangerau H (2013) Historical network analysis can be used to construct a social

447 network of 19th-century evolutionists. In: Fangerau H, Halling T, Geisler H, Martin W

448 (eds) Classification and Evolution in Biology, Linguistics and the History of Science:

449 Concepts - Methods - Visualization. Steiner, Stuttgart. pp. 45-65

450 Lapointe F-J, Legendre P (1995) Comparison tests for dendrograms: A comparative

451 evaluation. Journal of Classification 12:265-282

452 Le Bomin S, Lecointre G, Heyer E (2015) The evolution of musical diversity: The key role of

$453 \quad$ vertical transmission. PLOS One 11(3): e0151570

454 McIntosh R (1978) Matrix and plexus techniques. In: Whittaker RH (ed) Ordination of Plant

455 Communities, Junk, The Hague. pp.151-184

456 Morrison DA (2010) Using data-display networks for exploratory data analysis in

457 phylogenetic studies. Molecular Biology \& Evolution 27:1044-1057 
458 Morrison DA (2014) Phylogenetic networks — a new form of multivariate data summary for

459 data mining and exploratory data analysis. WIRES Data Mining Knowledge

$460 \quad$ Discovery 4:296-312

461 Najock D (1989) Computer-assisted reconstruction of texts. In: Bátori IS, Lenders W,

462 Putschke W (eds) Computational Linguistics, De Gruyter Berlin. pp. 534-544

463 O’Brien MJ, Darwent J, Lee Lyman R (2001) Cladistics is useful for reconstructing

464 archaeological phylogenies: Paleoindian points from the Southeastern United States.

465 Journal of Archaeological Science 28:1115-1136

466 Page RDM, Holmes EC (1998) Molecular Evolution. A Phylogenetic Approach. Blackwell, $467 \quad$ Oxford

468 Podani J (2000) Introduction to the Exploration of Multivariate Biological Data. Backhuys,

$469 \quad$ Leiden

470 Podani J (2001) SYN-TAX 2000. Computer Programs for Data Analysis in Ecology and

$471 \quad$ Systematics. Scientia, Budapest

472 Prieto JMC, Cabeza A, Aranda MÁG, Reina LL, Corrales M, Aguilar PC (2014) Las

473 figurillas de terracota del Teatro Romano de Málaga (España) Un estudio

474 arqueométrico. Boletín de la Sociedad Española de Cerámica y Vidrio 53:139-148

475 Rexová K, Frynta D, Zrzavy J. (2003) Cladistic analysis of languages: Indo-European

476 classification based on lexicostatistical data. Cladistics 19:120-127

477 Richards RJ (2015) Did Goethe and Schelling endorse species evolution? In: Lambier J,

478 Faflak J (eds) Marking Time: Romanticism and Evolution, University of Toronto 
Press, Toronto: (to appear). Accessed at:

480 http://home.uchicago.edu/ rjr6/Bibliographyl.htm

481 Rohlf FJ (1973) Hierarchical clustering using minimal spanning tree. Computer Journal 482 $16: 93-95$

Saitou N, Nei M (1987) The neighbor-joining method: A new method for reconstructing phylogenetic trees. Molecular Biology and Evolution 4:406-425

Sneath PHA, Sokal RR (1973) Numerical Taxonomy. $2^{\text {nd }}$ ed. Freeman, San Francisco Philosophy in the Nineteenth Century, Oxford University Press, Oxford. pp. 674-694

Swofford DL, Olsen GJ, Waddell PJ, Hillis DM (1996) Phylogenetic inference. In: Hillis DM, Moritz C, Mable BK (eds) Molecular Systematics, $2^{\text {nd }}$ edition, Sinauer, Sunderland. pp. $407-514$

\section{Electronic Appendix}

494 The data from Table 3 of Fisler and Lecointre (2013) rearranged to follow the three-cluster 495 classification obtained by UPGMA clustering (groups A to C), such that the sequence of 496 writings is in the same order as in the dendrogram of Fig. 2. Blocks of scores are emphasized 497 by rectangles in order to illustrate variables that have the highest explanatory power. 


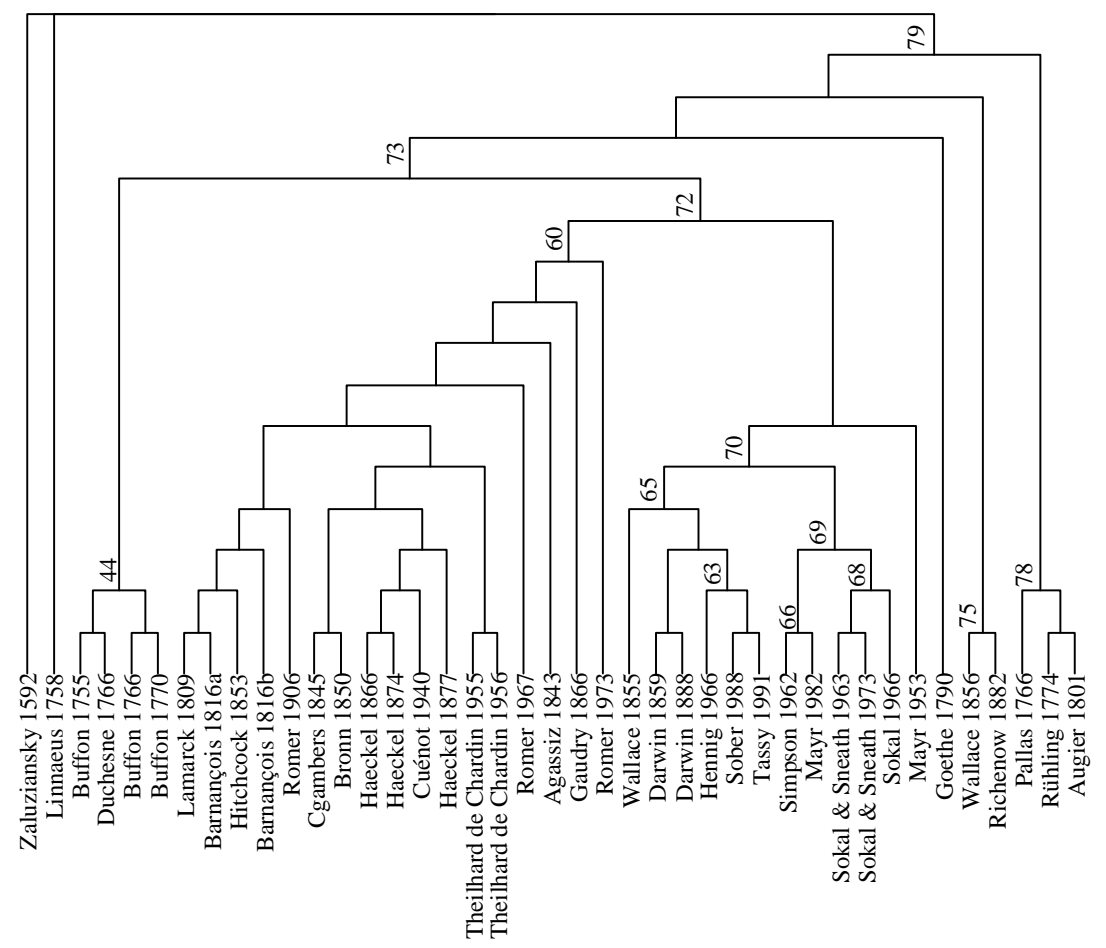

499

500 Fig. 1 Consensus cladogram (Tree 1) of 279 equally parsimonious trees (378 steps) for 41

501 writings on trees and classifications in systematics. The tree is not drawn to scale, and only

502 the sister group relations matter. Labels indicate "clades" recognized by the original authors

503 and referenced here as well. Modified from Fisler and Lecointre (2013) 


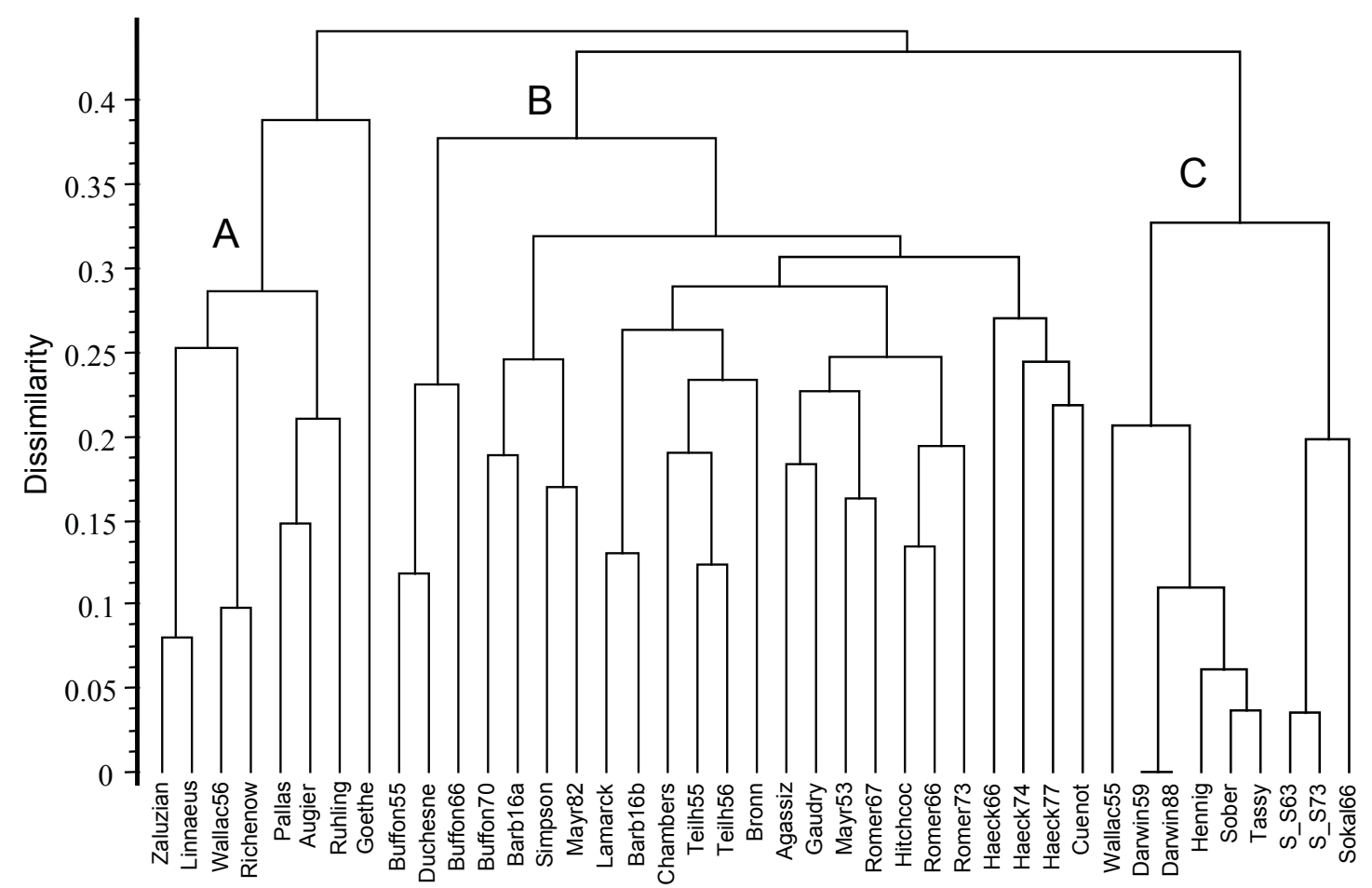

506 Fig. 2 Group average (UPGMA) clustering (Tree 2) based on Gower dissimilarities of 41

507 writings on trees and classifications in systematics. Original labels are trimmed to 8

508 characters, and are still self-explanatory (but see Figure 1 or Figure 6 for full names, as used

509 in FL). Letters identify three major groups, whereas Goethe remains as a singleton 


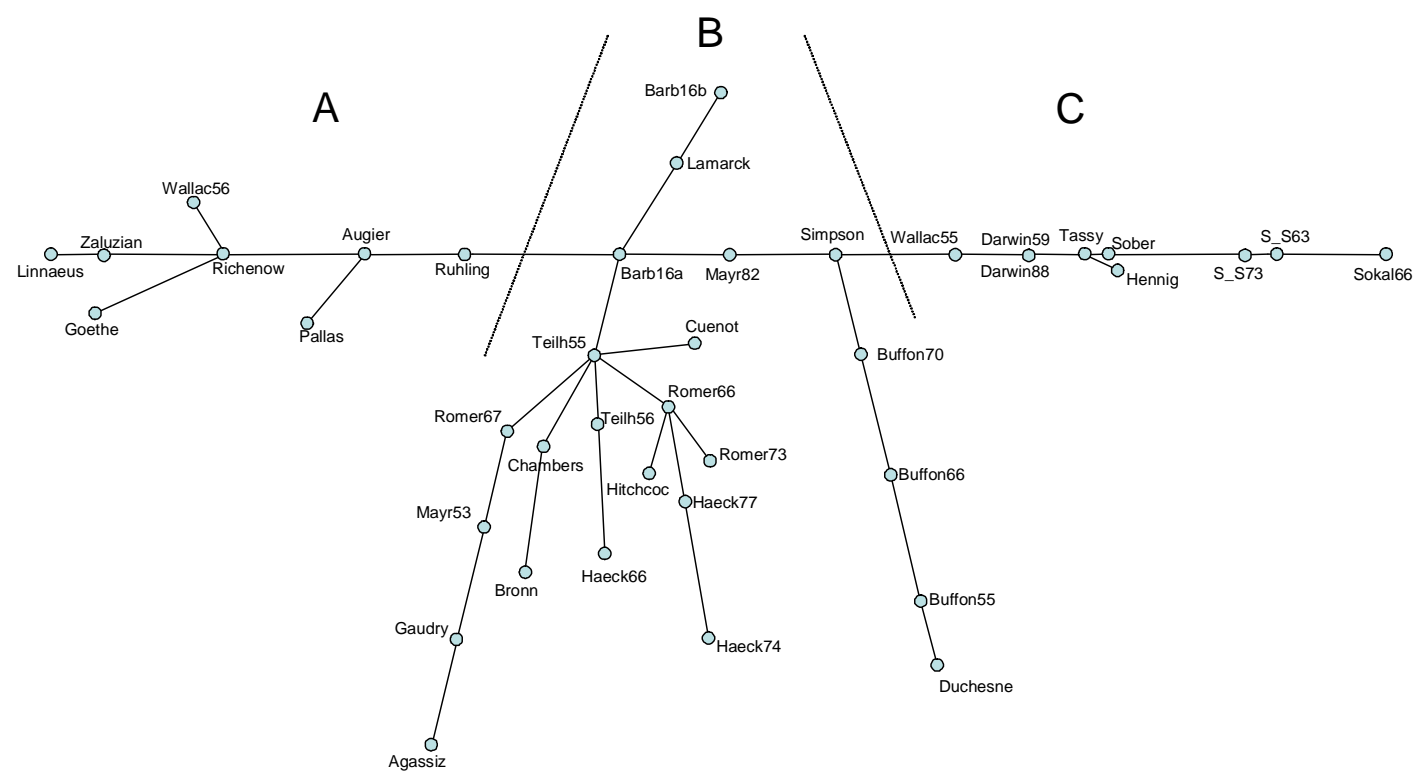

511

512 Fig. 3 Minimum spanning tree (Tree 3) based on Gower dissimilarities of 41 writings on trees

513 and classifications in systematics. Edge lengths are proportional to actual dissimilarities.

514 Letters identify three major groups separated by dotted lines. Labels as in Figure 1 


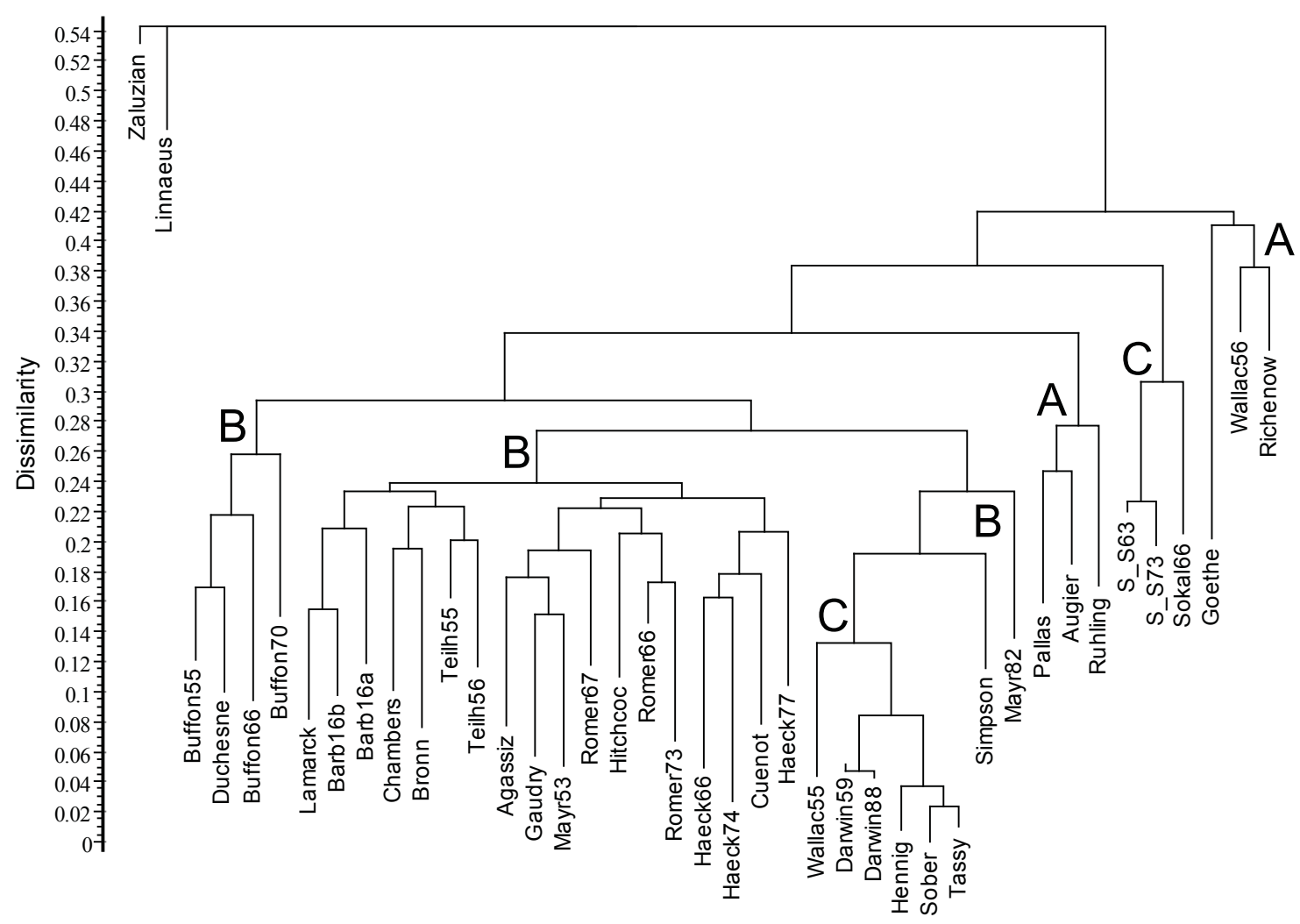

516

517 Fig. 4 Additive tree representation (Tree 4) of Gower dissimilarities between 41 writings on

518 trees and classifications in systematics. The outgroup is Zaluziansky. Letters refer to clusters

519 identified in Figure 2, as broken into several subtrees here 
bioRxiv preprint doi: https://doi org/10.1101/079483. this version posted January 132017 . The copyright holder for this preprint (which was not certified by peer review) is the author/funder, who has granted bioRxiv a license to display the preprint in perpetuity. It is made available under aCC-BY-NC-ND 4.0 International license.

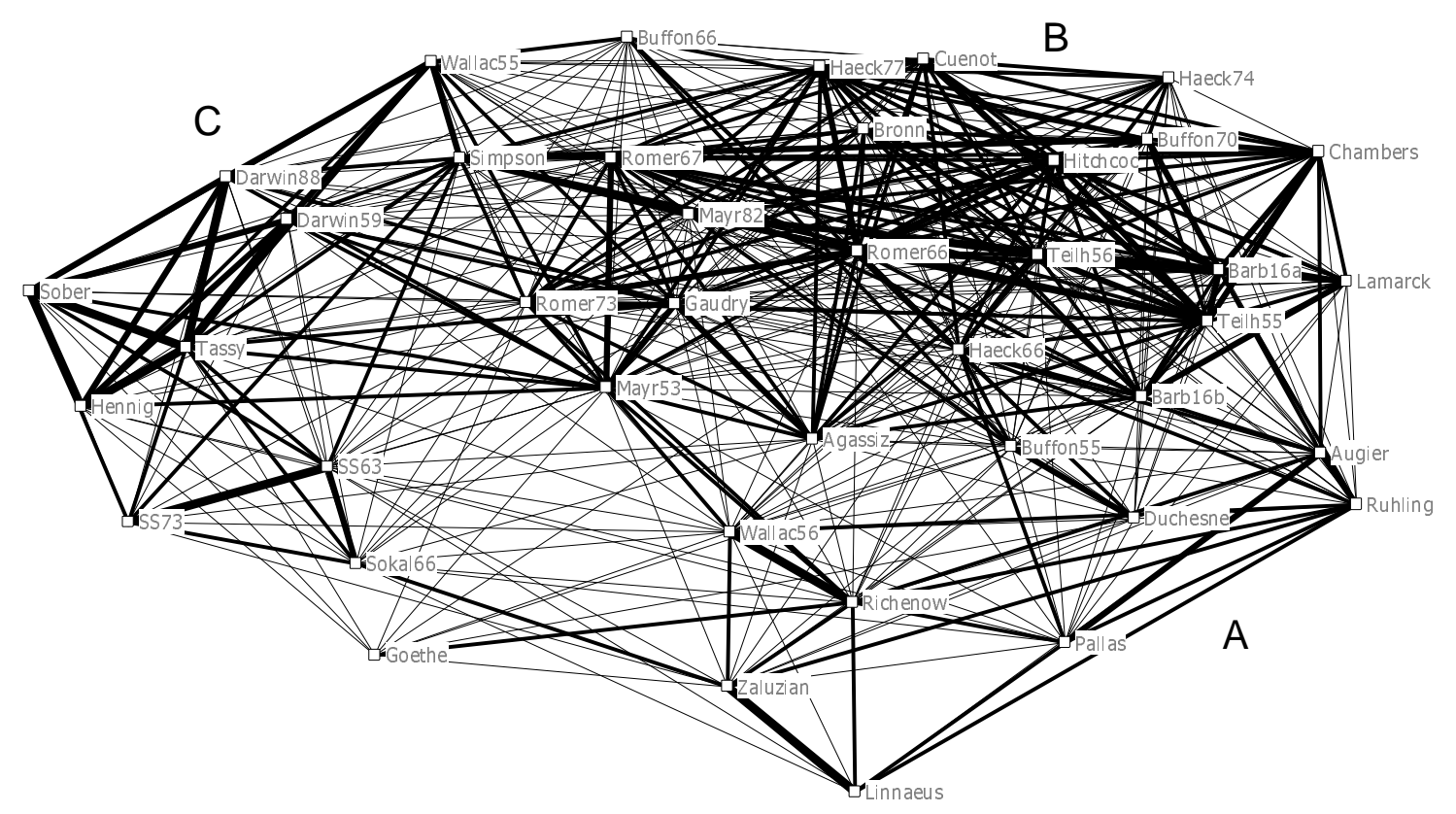

522 Fig. 5 Plexus graph based on Gower dissimilarities of 41 writings on trees and classifications

523 in systematics. See the text for an explanation of the line thickness categories. Letters refer to

524 clusters identified in Figure 2 


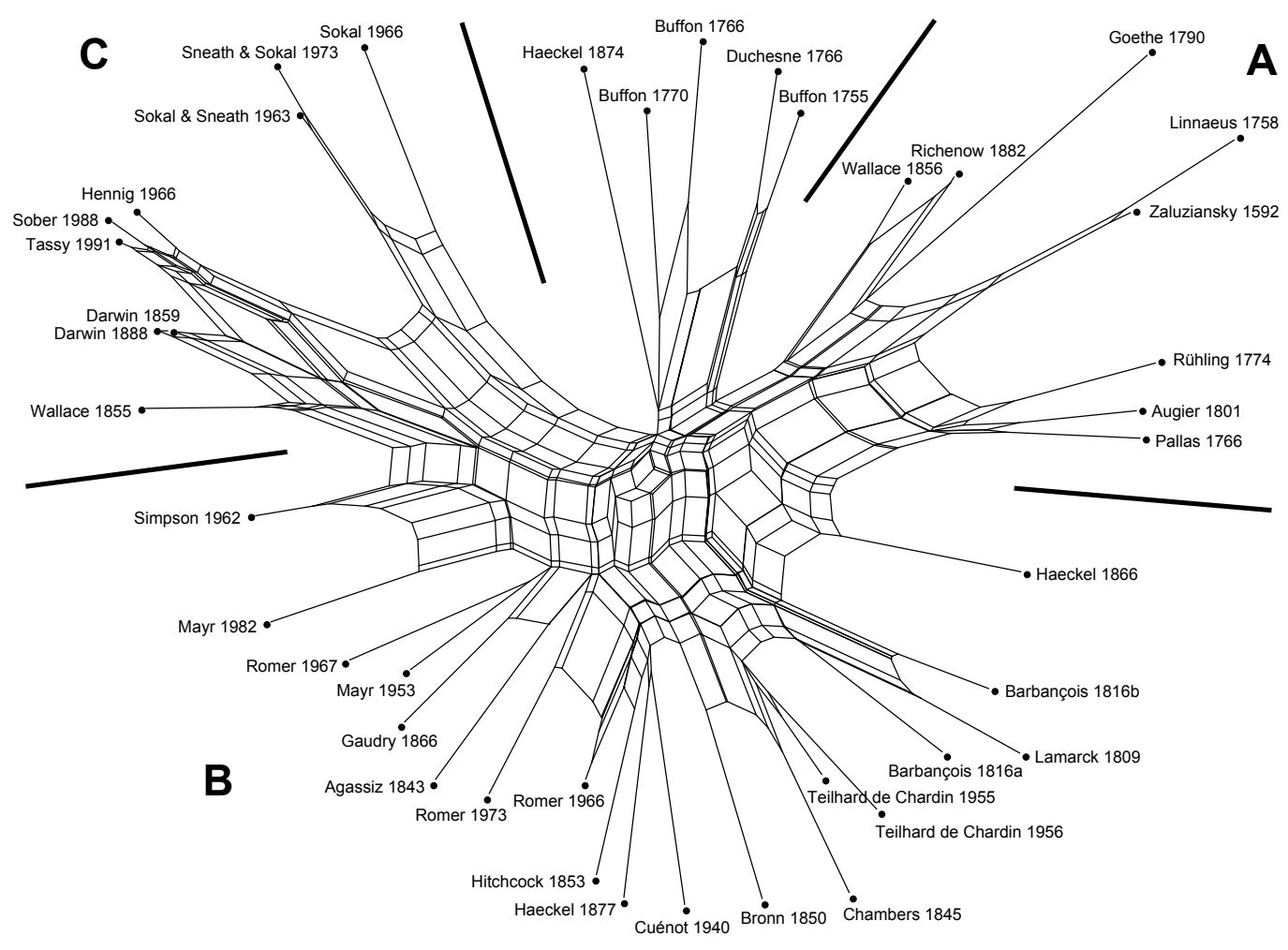

525

526 Fig. 6 Neighbor network based on Gower dissimilarities of 41 writings on trees and

527 classifications in systematics. Edge lengths are proportional to the original matrix distances.

528 Letters identify three major groups separated by solid lines. The original labels used in FL are

529 shown in full 


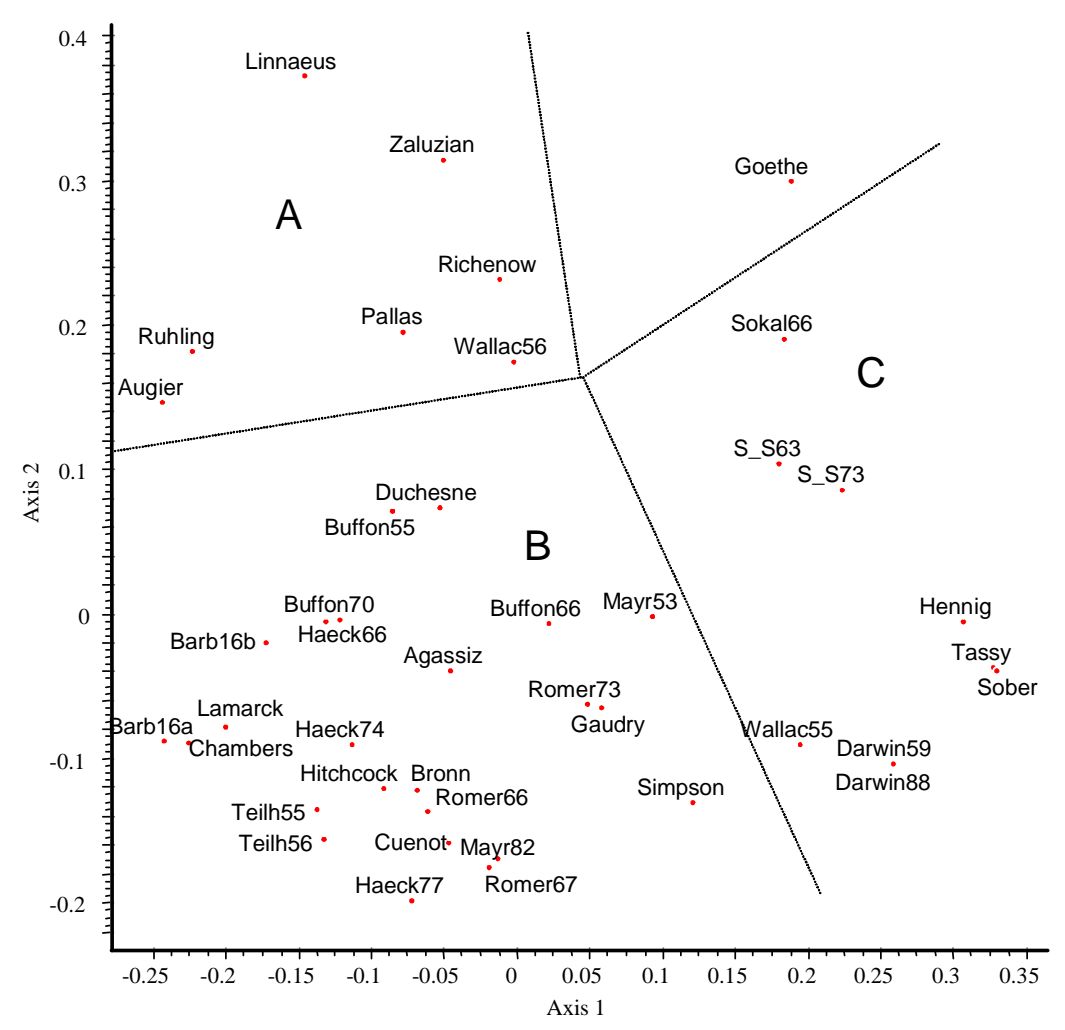

531

532 Fig. 7 Principal coordinates ordination based on Gower dissimilarities of 41 writings on trees

533 and classifications in systematics. Letters refer to clusters identified in Figure 2

534 


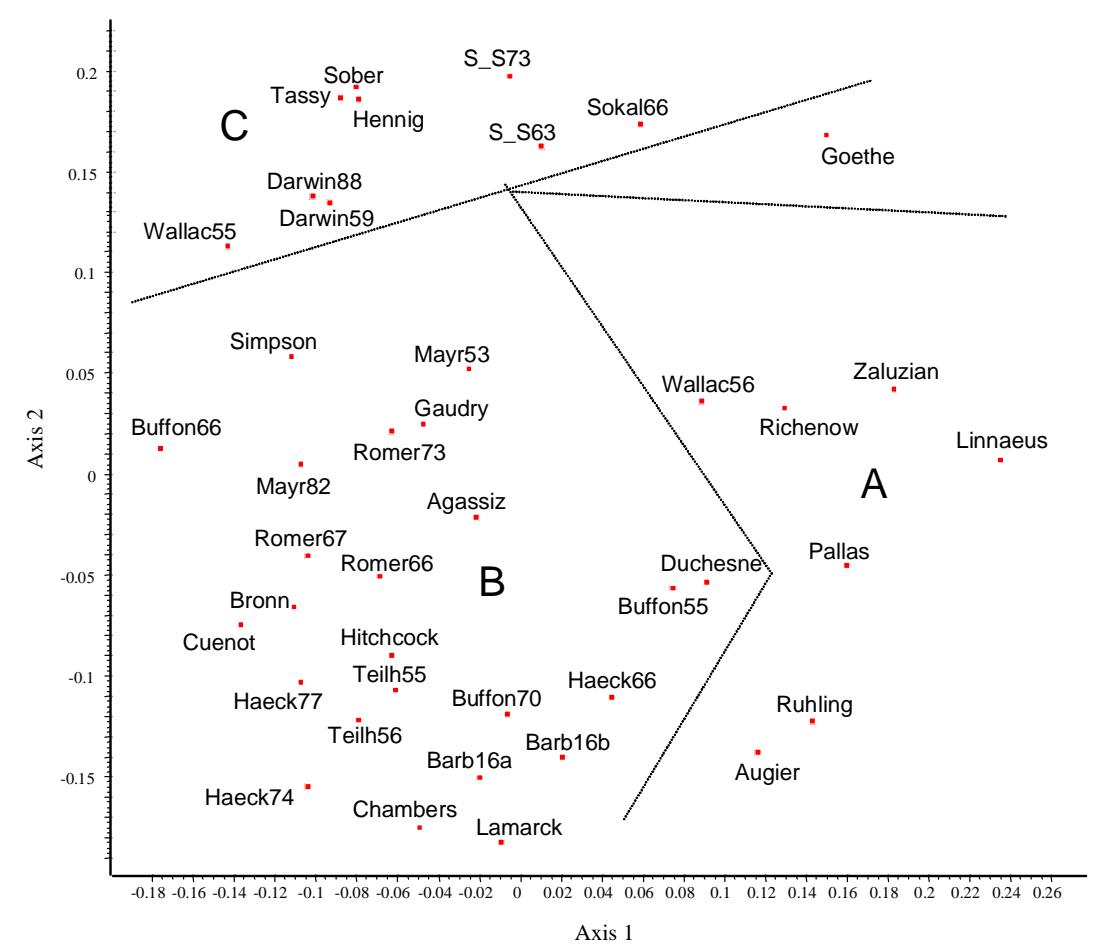

536 Fig. 8 Nonmetric multidimensional scaling ordination based on Gower dissimilarities of 41

537 writings on trees and classifications in systematics. Letters refer to clusters identified in

538 Figure 2; compare with the PCoA ordination in Figure 7

539 